mice have up to $30 \%$ fewer DRG neurons and $30 \%$ of DRG neurons normally express TrkB, the subset of neurons that fail to survive in the BDNF null mice might normally depend on an autocrine BDNF loop. Importantly, DRG neurons from the BDNF null mice were used to confirm that the effect of the antisense oligonucleotides depended on the expression of BDNF and that the oligonucleotides were not nonspecifically toxic. Taken together, these results indicate that a subset of adult sensory neurons can sustain their own survival in vitro, and probably also in vivo, by an autocrine loop. They also suggest that survival signalling continues to be essential for adult as well as developing cells ${ }^{14}$.

What of other kinds of neurons? Autocrine signalling may help to promote the survival of some developing neurons prior to target innervation ${ }^{11}$; furthermore, neurotrophins and their receptors are colocalized in a number of other types of neurons in the adult peripheral and central nervous systems. As Acheson et al. point out, these neuronal populations include those involved in amyotrophic lateral sclerosis, and Alzheimer's and Par- kinson's diseases, raising the possibility that failure of autocrine survival signalling could contribute to degenerative neurological disease. Lastly, it will be of great interest to find out whether the types of adult neurons that are normally able to promote their own survival by an autocrine mechanism are better able to survive and regenerate their axons after injury.

The findings of Acheson et al. suggest a simple explanation as to why developing sensory neurons switch from a targetderived to an autocrine supply of survival factors. In accord with the neurotrophic hypothesis, a competition for targetderived survival signals may initially help to match the numbers of neurons and target cells. The survival of the winning neurons may then be stabilized in adulthood by autocrine mechanisms to ensure their preservation for the lifetime of the organism; glial cells, which develop for the most part after target innervation, may provide additional survival factors (ref. 15; A. Meyer-Franke and B. Barres, unpublished observations).

If adult sensory neurons do not need target-derived neurotrophic signals to survive, what do such signals do in the adult? The answer is probably that they act locally to regulate the survival and plasticity of axon terminals ${ }^{16,17}$. Consistent with this view, anti-NGF antibodies prevent collateral sprouting but do not induce death of adult DRG neurons ${ }^{17}$.

The relative importance of autocrine and target-derived survival signalling in the nervous system may not become clear for some time, but some basic mechanistic questions can be addressed in the near future. For instance, does the autocrine ligand/receptor interaction require that the ligand is first secreted, or can it act intracellularly? Can a cell regulate the type of interactions that occur by preferentially sorting ligands or receptors to specific cellular locations? And can electrical activity regulate the autocrine loop ${ }^{18}$ ? Whatever the answers, this much is already clear: unlike good marksmanship, understanding neuronal survival may sometimes require focusing away from the target.

John V. Heymach Jr and Barbara A. Barres are in the Department of Neurobiology, Stanford University School of Medicine, Stanford, California 94305, USA.

\title{
William Alfred Fowler (1911-95)
}

WiLliam Alfred Fowler, who shared the 1983 Nobel prize for physics, died on 14 March in Pasadena, California, at the age of 83 . He received his undergraduate education at Ohio State University, mov. ing in 1936 as a graduate student to the California Institute of Technology, where he spent the rest of his research career.

Fowler began his career by measuring beta-ray spectra with the aim of determining the form of the weak nuclear interaction. This led naturally, after the end of the Second World War, to determining experimental cross-sections for nuclear reactions of interest to astrophysics, a field in which for many years the Kellogg Radiation Laboratory at the California Institute of Technology played a unique role.

In a monumental sequence of papers, Fowler and his colleagues in the Kellogg Radiation Laboratory provided the essential experimental basis for the processes known nowadays as hydrogenburning, the P-P chain (both these processes are catalytic cycles that convert hydrogen to helium) and the carbonnitrogen cycle, work that was cited in the Nobel prize award of later years.

From 1953 onwards, Fowler turned his attention increasingly to the great com. plex of nuclear reactions that characterize the later stages of stellar evolution. Together with Geoffrey and Margaret Burbidge and myself, he was co-author in 1957 of a seminal paper in this field, a paper which became familiarly known as $B^{2} F H$. The paper showed how by nuclear reactions in stars, occurring at increas. ing temperatures throughout stellar evolution, it was possible to build all

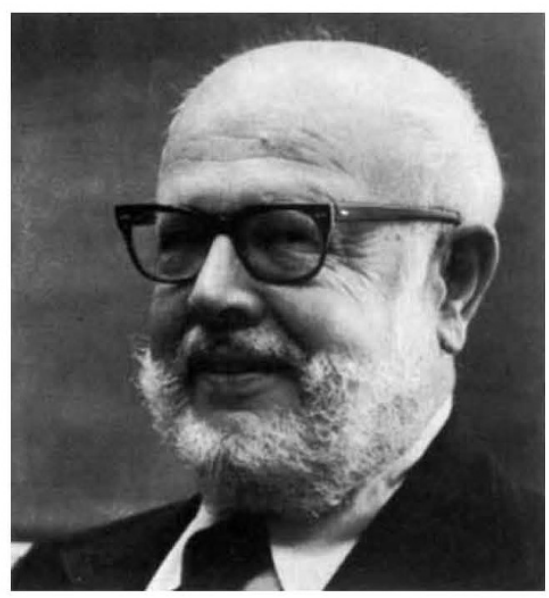

Willy Fowler, who died this month.

the chemical elements starting from hydrogen. Broadly speaking, the lighter elements and the transition elements up to nickel were synthesized in charged particle reactions, and heavier elements, from relative atomic mass about 60 upwards, in neutron reactions, although nuclides of low natural abundance often departed from this simple model. He and I developed a number of spin-offs from $\mathrm{B}^{2} \mathrm{FH}$ in later years. These included in
1960 the first steps of what became known as nuclear chronology, a topic in which Fowler always remained active down the years. In 1964 came the theory of supernovae, with an attempt to combine the ideas on nucleosynthesis of $B^{2} \mathrm{FH}$ with the astrophysical evolution of supernovae up to the moment of their explosion. In 1967, in collaboration with R. V. Wagoner and myself, he developed what became known as Big Bang nucleosynthesis; and in 1975 he was the senior author, in collaboration with E. R. Caughlin and B. A. Zimmerman, of another far-ranging work on thermonuclear reactions in stars. This provided basic information on nuclear reaction rates that was encyclopaedic for almost all reactions up to relative atomic mass about 25, an immense improvement on what had been available at the commencement of Fowler's work.

The technical description of a man's career unfortunately says little of what he was really like. Known everywhere as 'Willy', there can have been few scientists who have been so widely liked on the entire international stage. $\mathrm{He}$ will be greatly missed.

Willy Fowler is survived by his second wife, Mary Dutcher Fowler, and his two daughters by his first wife. Fred Hoyle

Sir Fred Hoyle was formerly the Director of the Institute of Theoretical Astronomy, Cambridge, UK. 